\title{
Constraining dynamical dark energy with a divergence-free parametrization in the presence of spatial curvature and massive neutrinos
}

\author{
Hong $\mathrm{Li}^{1,2}$ and Xin Zhang*3,4,† \\ ${ }^{1}$ Institute of High Energy Physics, Chinese Academy of Sciences, Beijing 100049, China \\ ${ }^{2}$ National Astronomical Observatories, Chinese Academy of Sciences, Beijing 100012, China \\ ${ }^{3}$ Department of Physics, College of Sciences, Northeastern University, Shenyang 110004, China \\ ${ }^{4}$ Center for High Energy Physics, Peking University, Beijing 100080, China
}

\begin{abstract}
In this paper, we report the results of constraining the dynamical dark energy with a divergence-free parameterization, $w(z)=w_{0}+w_{a}\left(\frac{\ln (2+z)}{1+z}-\ln 2\right)$, in the presence of spatial curvature and massive neutrinos, with the 7-yr WMAP temperature and polarization data, the power spectrum of LRGs derived from SDSS DR7, the Type Ia supernova data from Union2 sample, and the new measurements of $H_{0}$ from HST, by using a MCMC global fit method. Our focus is on the determinations of the spatial curvature, $\Omega_{k}$, and the total mass of neutrinos, $\sum m_{v}$, in such a dynamical dark energy scenario, and the influence of these factors to the constraints on the dark energy parameters, $w_{0}$ and $w_{a}$. We show that $\Omega_{k}$ and $\sum m_{v}$ can be well constrained in this model; the 95\% CL limits are: $-0.0153<\Omega_{k}<0.0167$ and $\sum m_{v}<0.56 \mathrm{eV}$. Comparing to the case in a flat universe, we find that the error in $w_{0}$ is amplified by $25.51 \%$, and the error in $w_{a}$ is amplified by $0.14 \%$; comparing to the case with a zero neutrino mass, we find that the error in $w_{0}$ is amplified by $12.24 \%$, and the error in $w_{a}$ is amplified by $1.63 \%$.
\end{abstract}

PACS numbers: 95.36.+x, 98.80.Es, 98.80.-k

\section{INTRODUCTION}

Dark energy is one of the most important themes in physics today. However, we do not know much about dark energy due to the accuracy of current data. Though the current observational data are consistent with a cosmological constant, the possibility that dark energy is dynamical is still not excluded by the data and has been attracting wide attentions in the cosmology and theoretical physics communities.

In order to detect the dynamics of dark energy, one usually has to parameterize the equation-of-state parameter (EOS), $w$, empirically, using two or more free parameters. Among all the parametrization forms of EOS, the Chevallier-PolarskiLinder (CPL) parametrization [1], $w(a)=w_{0}+w_{a}(1-a)$, where $w_{0}$ and $w_{a}$ are parameters and $a$ is the scale factor of the universe, is the most widely used one and has been explored extensively. However, as pointed out in Ref. [2], the CPL description will lead to unrealistic behavior in the future evolution, i.e., $|w(z)|$ grows rapidly and eventually encounters divergence as the redshift $z$ approaches -1 . In order to keep the advantage of the CPL parametrization and avoid its drawback at the same time, it is believed that a parametrization that is free of divergence both in the past and future evolutions is necessary.

In Ref. [2], Ma and Zhang proposed the following hybrid form of logarithm and CPL parametrizations:

$$
w(z)=w_{0}+w_{a}\left(\frac{\ln (2+z)}{1+z}-\ln 2\right) .
$$

This novel parametrization has well behaved, bounded evolution for both high redshifts and negative redshifts. In particular, for the limiting situation, $z \rightarrow-1$, a finite value for EOS

\footnotetext{
${ }^{*}$ Corresponding author

†Electronic address: zhangxin@mail.neu.edu.cn
}

can be obtained, $w=w_{0}+w_{a}(1-\ln 2)$. At low redshifts, this parametrization form reduces to the linear one, $w(z) \approx$ $w_{0}+\tilde{w}_{a} z$, where $\tilde{w}_{a}=-(\ln 2) w_{a}$. Of course, one can also recast it at low redshifts as the CPL form, $w(z) \approx w_{0}+\tilde{w}_{a} z /(1+z)$, where $\tilde{w}_{a}=(1 / 2-\ln 2) w_{a}$. Therefore, it is clear to see that this parametrization exhibits well-behaved feature for the dynamical evolution of dark energy. Without question, such a two-parameter form of EOS can genuinely cover scalar-field models as well as other theoretical scenarios. In Ref. [3], this parametrization was used to explore the ultimate fate of the universe.

The parametrization (1) has been explored deeply by using the current data. In particular, in Ref. [4], we have analyzed the detection of dynamics of dark energy with the parametrization (1) by performing a Markov Chain Monte Carlo (MCMC) global fitting method. Since dark energy parameters are tightly correlated with the spatial curvature $\Omega_{k}$ and neutrino mass $\sum m_{v}$, in this paper we will deeply analyze the influences of these factors on the detection of dynamics of dark energy.

The paper is organized as follows: In Sec. II we will introduce our global fitting procedure and the data we used for analysis; the results are presented in Sec. III, and our conclusion is given in Sec. IV.

\section{GLOBAL FITTING PROCEDURE AND DATA}

We have modified MCMC package CosmoMC [5] to perform a global fitting analysis for the dynamical dark energy parameterized above. As we know, within the dynamical dark energy models, the perturbations of dark energy are important [6-8] for the data fitting analysis. For quintessencelike or phantomlike models, whose $w$ does not cross the cosmological constant boundary, the perturbation of dark energy is well defined. However, when $w$ crosses -1 , which is described by a quintom dark energy model [9], one is encountered with 
the divergence problem for perturbations of dark energy at $w=-1$. For avoiding such kind of divergence problem, in this paper we use the method provided in Refs. $[8,10]$ to treat the dark energy perturbations consistently in the whole parameter space in the numerical calculations.

Our most general parameter space vector is:

$$
\mathbf{P} \equiv\left(\omega_{b}, \omega_{c}, \Theta, \tau, w_{0}, w_{a}, \Omega_{k}, \sum m_{v}, n_{s}, A_{s}, c_{s}^{2}\right),
$$

where $\omega_{b} \equiv \Omega_{b} h^{2}$ and $\omega_{c} \equiv \Omega_{c} h^{2}$, with $\Omega_{b}$ and $\Omega_{c}$ the physical baryon and cold dark matter densities relative to the critical density, $\Omega_{k}$ is the spatial curvature satisfying $\Omega_{k}+\Omega_{m}+\Omega_{\mathrm{de}}=$ $1, \sum m_{v}$ is the total mass of neutrinos, $\Theta$ is the ratio (multiplied by 100) of the sound horizon to the angular diameter distance at decoupling, $\tau$ is the optical depth to re-ionization, $w_{0}$ and $w_{a}$ are the parameters of dark energy EOS given by Eq. (1), $A_{s}$ and $n_{s}$ are the amplitude and the spectral index of the primordial scalar perturbation power spectrum, and $c_{s}$ is the sound speed of dark energy. For the pivot scale we set $k_{s 0}=0.05 \mathrm{Mpc}^{-1}$. Note that we have assumed purely adiabatic initial conditions.

Note that the sound speed of dark energy is fixed in our analysis. In the framework of the linear perturbation theory, besides the EOS of dark energy, the dark energy perturbations can also be characterized by the sound speed, $c_{s}^{2} \equiv \delta p_{\mathrm{de}} / \delta \rho_{\mathrm{de}}$. The sound speed of dark energy might affect the evolution of perturbations, and might leave signatures on the CMB power spectrum [11]. However, it has been shown that the constraints on the dark energy sound speed $c_{s}^{2}$ in dynamical dark energy models are still very weak, since the current observational data are still not accurate enough [12]. Therefore, in our analysis, we have treated the dark energy as a scalar-field model (multi-fields or single field with high derivative) and set $c_{s}^{2}$ to be 1 . Of course, one can also take $c_{s}^{2}$ as a parameter, but the fit results would not be affected by this treatment [12].

In the computation of the cosmic microwave background anisotropy (CMB), we include the 7-year WMAP temperature and polarization power spectra [13] with the routine for computing the likelihood supplied by the WMAP team [14]. For the large scale structure (LSS) information, we use the power spectrum of luminous red galaxies (LRGs) measured from the SDSS DR7 [15]. The supernova (SN) data we use are the recently released "Union2" sample of 557 data [16]; note that the systematic errors are included in our analysis. In the calculation of the likelihood from SN we marginalize over the relevant nuisance parameter [17].

Furthermore, we make use of the Hubble Space Telescope (HST) measurement of the Hubble constant $H_{0} \equiv$ $100 h \mathrm{~km} \mathrm{~s}^{-1} \mathrm{Mpc}^{-1}$ by a Gaussian likelihood function centered around $h=0.738$ and with a standard deviation $\sigma=$ 0.024 [16].

\section{NUMERICAL RESULTS}

In this section we shall present the results of the global fitting analysis. In particular, we shall test the influences on probing the dynamics of dark energy when spatial curvature and massive neutrinos are considered in the data analysis. Since we know that both the spatial curvature parameter $\Omega_{k}$ and the total mass of neutrinos $\sum m_{v}$ are degenerate with the equation of state of dark energy $w$, we are very much interested in seeing how these two factors affect the constraints on the parameters $w_{0}$ and $w_{a}$. Moreover, we also want to see how the current data constrain $\Omega_{k}$ and $\sum m_{v}$ within the framework of dynamical dark energy with parametrization (1).

\section{A. Spatial curvature and EOS}

Dark energy parameters and $\Omega_{k}$ are correlated via the cosmological distance information. From the observation of $\mathrm{CMB}$, the curvature of the observable universe $\Omega_{k}$ can be determined by the position of first acoustic peak of CMB temperature power spectrum precisely. However, $\Omega_{k}$ is tightly degenerated with $\Omega_{m}$, and such degeneracy can be, in certain level, broken by taking into account the data of large scale structure and supernovae.

In constraining $\Omega_{k}$, one usually uses a combination of distance measurements from baryon acoustic oscillations (BAO) and CMB. In this paper, however, we use the power spectrum of LRGs measured from SDSS DR7, instead of BAO, to constrain $\Omega_{k}$. Note that BAO and the LRG power spectrum cannot be treated as independent data sets because a part of the measurement of BAO comes from LRGs as well. Since the LRG power spectrum is a powerful probe of the total mass of neutrinos, $\sum m_{v}$, in this paper we uniformly use the LRG power spectrum, instead of BAO, to constrain $\Omega_{k}$ and $\sum m_{v}$. In the following we shall consider the case of massless neutrinos and see the influence of $\Omega_{k}$.

In Fig. 1, we present the two dimensional cross correlation and one dimensional probability distribution of $\Omega_{k}, w_{0}, w_{a}$, $\Omega_{m}$ and $H_{0}$ by fitting with 7-yr WMAP, LRG, SN and $H_{0}$ data. From the panels of $\Omega_{k}-w_{0}$ and $\Omega_{k}-w_{a}$, we find that the correlation between $\Omega_{k}$ and $w_{0}$ is positive, but we do not observe much correlation between $\Omega_{k}$ and $w_{a}$.

The $68 \%$ confidence level (CL) constraint on $\Omega_{k}$ is $0.0003 \pm$ 0.0079 , from which we can see that our universe is very close to flatness. The $95 \% \mathrm{CL}$ limit is: $-0.0153<\Omega_{k}<0.0167$. So, we find that in the dynamical dark energy model (1) the spatial curvature is well constrained by WMAP $+\mathrm{LRG}+\mathrm{SN}+H_{0}$. It is of interest to make a comparison to the cases of $\Lambda \mathrm{CDM}(w=$ -1 ) model and constant $w$ model. Komatsu et al. gave the limit on $\Omega_{k}$ for the case of $w=-1, \Omega_{k}=-0.0023_{-0.0056}^{+0.0054}(68 \%$ $\mathrm{CL}$ ), from $\mathrm{WMAP}+\mathrm{BAO}+H_{0}$, and for the case of constant $w, \Omega_{k}=-0.0057_{-0.0068}^{+0.0066}(68 \% \mathrm{CL})$, from $\mathrm{WMAP}+\mathrm{BAO}+\mathrm{SN}$, where $\mathrm{SN}$ is the Constitution sample. These results are in good agreement with our limit.

How does the spatial curvature parameter, $\Omega_{k}$, affect the constraint results of $\left(w_{0}, w_{a}\right)$ ? We find that, in a non-flat universe, the results are:

$$
w_{0}=-0.922 \pm 0.123 \text { and } w_{a}=1.651 \pm 1.470(68 \% \mathrm{CL}) .
$$

Thus, even when $w$ is allowed to depend on time, the current data are still consistent with a cosmological constant. 

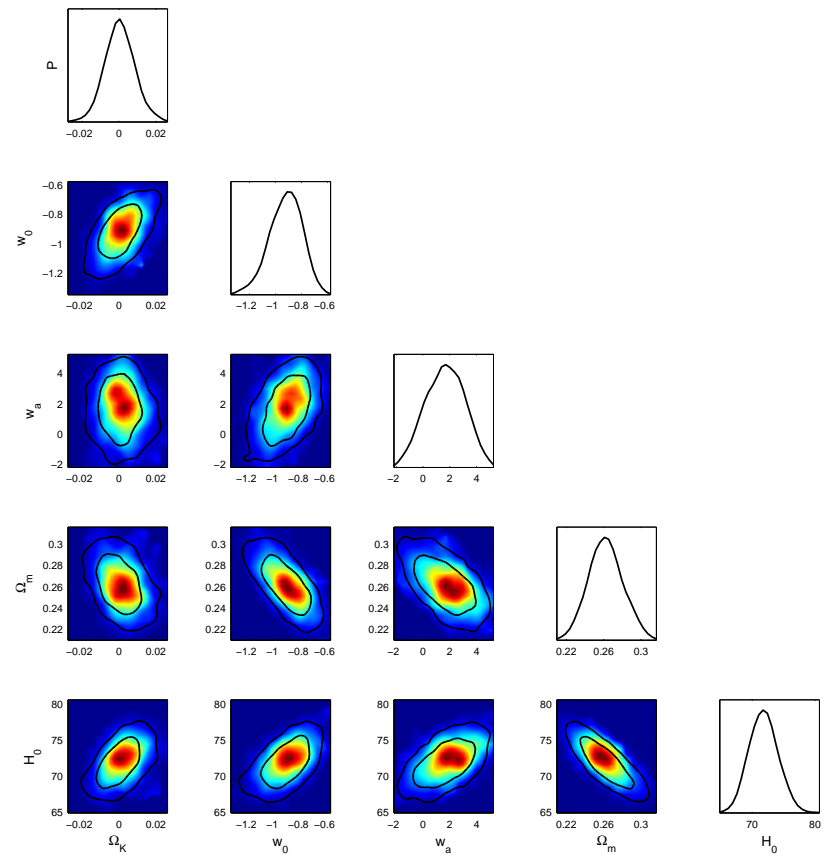

FIG. 1: Constraints on the cosmological parameters $\Omega_{k}, w_{0}, w_{a}, \Omega_{m}$ and $H_{0}$ in the dynamical dark energy scenario (1).

However, a large range of values of $\left(w_{0}, w_{a}\right)$ are still allowed by the data. Comparing to the case in a flat universe: $w_{0}=-0.921 \pm 0.098$ and $w_{a}=1.905 \pm 1.468(68 \% \mathrm{CL})$, we find that the best-fit values of $w_{0}$ and $w_{a}$ are shifted by $0.1 \%$ and $13.3 \%$, respectively; the error in $w_{0}$ is amplified by $25.51 \%$, and the error in $w_{a}$ is almost the same, only amplified by $0.14 \%$.

One may be curious to know why the error on $w_{0}$ is significantly amplified, but that for $w_{a}$ is scarcely affected by the presence of $\Omega_{k}$. Actually, this is fairly easy to be understood. From the fitting results, it is clear to see that $w_{0}$ is constrained more tightly than $w_{a}$, i.e., $w_{0}$ is more sensitive to the data, comparing to $w_{a}$. The current constraint on $w_{a}$ is very weak, only at the level of $\sim 80 \%$, while for $w_{0}$ the constraint is much stronger, at the level of $\sim 10 \%$. Thus, once $\Omega_{k}$ is involved in the analysis, its influence on $w_{0}$ is, obviously, much greater than that on $w_{a}$.

Of course, the fact that $w_{a}$ is insensitive to the data is not specific to our parametrization form. In general, for a twoparameter dark energy EOS form in which $w_{0}$ serves as a constant part and $w_{a}$ is used to describe the dynamical evolution of the EOS, $w_{a}$ is always insensitive to the data comparing to $w_{0}$. The constraints on $w_{0}$ and $w_{a}$ are mainly determined from their contributions to the Hubble expansion rate $H(z)$ which is more sensitive to $w_{0}$ than $w_{a}$. During the calculation, considering the same variations of $w_{0}$ and $w_{a}$, the effect on $H(z)$ from $w_{0}$ is more significant than that from $w_{a}$. Thus, when using the observational data to explore dark energy EOS, the constraint on $w_{0}$ is usually better than that on $w_{a}$ by about one order of magnitude. This explains the fact that the timeevolution of dark energy EOS cannot be well probed by the current cosmological data. Taking the number of parameters into account, it has been shown that the $\Lambda \mathrm{CDM}$ model is still the best one among all the current dark energy models; see, e.g., Refs. [18, 19]. It is expected that the dark energy observations of the next generation could measure the dynamical evolution of dark energy accurately.

So, our conclusion that $w_{0}$ is affected by $\Omega_{k}$ more severely than $w_{a}$ is universal, not specific to the parametrization adopted in this paper. Obviously, the same conclusion is applicable to the case of massive neutrinos that will be discussed in the next subsection.

\section{B. Neutrino mass and EOS}

Weighting the neutrino mass is one of the most important challenges in modern physics. Currently the neutrino oscillation experiments, such as atmospheric neutrinos experiments [20] and solar neutrinos experiments [21], have confirmed that the neutrinos are massive, but give no hint on their absolute mass scale. Cosmology can provide crucial complementary information on absolute neutrino masses, because massive neutrinos leave imprints on the cosmological observations, such as the Hubble diagram, CMB temperature power spectrum and LSS matter power spectrum [22].

In Ref. [13], Komatsu et al. have constrained the total mass of neutrinos, $\sum m_{v}=94 \mathrm{eV}\left(\Omega_{v} h^{2}\right)$, from the 7-yr WMAP data combined with the distance information. For a flat $\Lambda$ CDM model, i.e., $w=-1$ and $\Omega_{k}=0$, they found that the WMAP+BAO $+H_{0}$ limit is $\sum m_{v}<0.58 \mathrm{eV}(95 \%$ $\mathrm{CL}$ ). The limit improves when information on the growth of structure is added. For example, they found that, when the $\mathrm{BAO}$ is replaced by the power spectrum of LRGs, the combination WMAP $+\mathrm{LRG}+H_{0}$ gives $\sum m_{v}<0.44 \mathrm{eV}(95 \% \mathrm{CL})$ for $w=-1$. Thus, we can see that the power spectrum of LRGs plays an important role in constraining the neutrino mass. In the following we shall use the combination of $\mathrm{WMAP}+\mathrm{LRG}+\mathrm{SN}+H_{0}$ to constrain the neutrino mass, $\sum m_{v}$, as well as the parameters of dynamical dark energy, $w_{0}$ and $w_{a}$, in a flat universe.

In Fig. 2, we present the two dimensional cross correlation and one dimensional probability distribution of $\sum m_{v}, w_{0}, w_{a}, \Omega_{m}$ and $H_{0}$, from the data combination $\mathrm{WMAP}+\mathrm{LRG}+\mathrm{SN}+H_{0}$. From the panels of $\sum m_{v}-w_{0}$ and $\sum m_{v}-w_{a}$, we find that there is an anti-correlation between $w_{0}$ and $\sum m_{v}$, but no significant correlation between $w_{a}$ and $\sum m_{v}$ is observed.

The global fitting gives the constraint on the neutrino mass in our dynamical dark energy scenario: $\sum m_{v}<0.56 \mathrm{eV}(95 \%$ CL). So, it is interesting to find that in our dynamical dark energy scenario (1) the total mass of neutrinos can be well constrained by the data combination WMAP+LRG $+\mathrm{SN}+\mathrm{H}_{0}$. To make a comparison, we show the results of the constant $w$ model given by Komatsu et al. [13]: $\sum m_{v}<0.71 \mathrm{eV}(95 \%$ CL) from WMAP+LRG $+H_{0}$, and $\sum m_{v}<0.91 \mathrm{eV}(95 \% \mathrm{CL})$ from WMAP+BAO+SN (where $\mathrm{SN}$ is the Constitution data). In our case, even though there is one more parameter, $w_{a}$, the constraint on $\sum m_{v}$ is much tighter. This shows that the data 

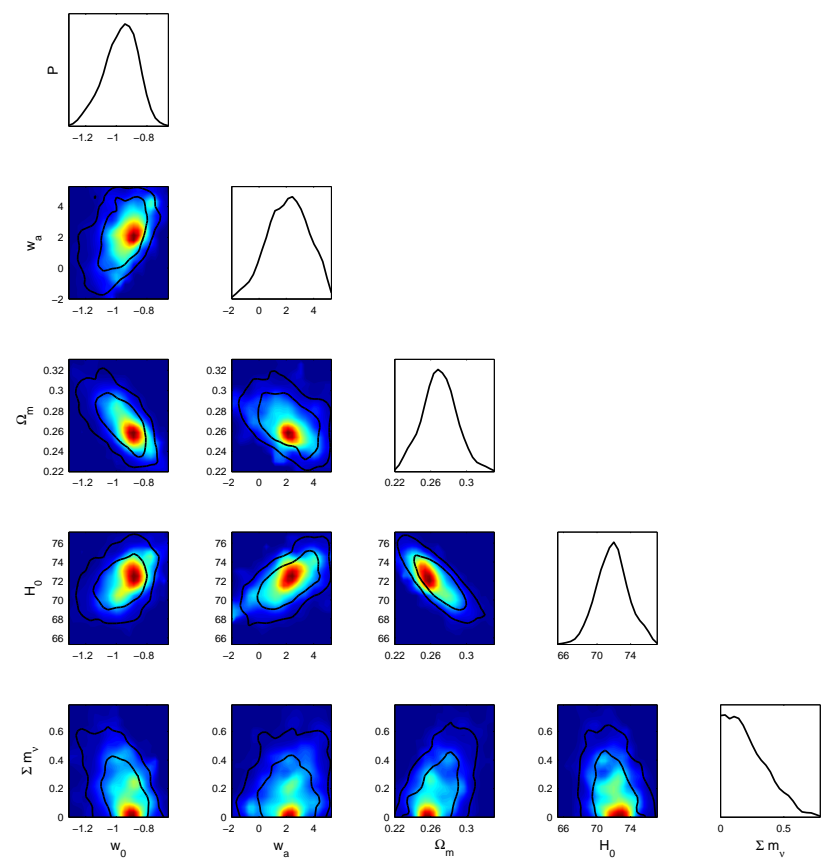

FIG. 2: Constraints on the cosmological parameters $w_{0}, w_{a}, \Omega_{m}, H_{0}$ and $\sum m_{v}$ in the dynamical dark energy scenario (1).

combination we used, WMAP+LRG $+\mathrm{SN}+\mathrm{H}_{0}$, is fairly good at constraining the neutrino mass. To show this more clearly, we also use our data combination to make a global fitting calculation for the case of the constant $w$ model, and the constraint result we obtained is $\sum m_{v}<0.45 \mathrm{eV}$ (95\% CL).

How does the neutrino mass, $\sum m_{v}$, affect the constraint results of $\left(w_{0}, w_{a}\right)$ ? We find that, with the non-zero neutrino mass, the results are:

$$
w_{0}=-0.972 \pm 0.110 \text { and } w_{a}=2.038 \pm 1.492(68 \% \mathrm{CL}) .
$$

So, still, though the current data are consistent with a cosmological constant, a large range of values of $\left(w_{0}, w_{a}\right)$ are still allowed by the data. Comparing to the case with a zero neutrino mass, $w_{0}=-0.921 \pm 0.098$ and $w_{a}=1.905 \pm 1.468(68 \%$ $\mathrm{CL}$ ), we find that the best-fit value of $w_{0}$ is shifted by $5.5 \%$, and the best-fit value of $w_{a}$ is shifted by $7.0 \%$; the error in $w_{0}$ is amplified by $12.24 \%$, and the error in $w_{a}$ is amplified by $1.63 \%$. Obviously, the reason that the influence on the error of $w_{0}$ by the presence of $\sum m_{v}$ is much severer than that of $w_{a}$ is as the same as the case of the spatial curvature: $w_{0}$ is much more sensitive to the data than $w_{a}$.

\section{CONCLUSION}

With the 7-yr WMAP temperature and polarization data, the power spectrum of LRGs derived from SDSS DR7, the Type Ia supernova data from Union2 sample, and the new measurements of $H_{0}$ from HST, we have tested the dynamical dark energy parametrization, $w(z)=w_{0}+w_{a}\left(\frac{\ln (2+z)}{1+z}-\ln 2\right)$, in the presence of spatial curvature and massive neutrinos, by using a MCMC global fit method. For a time-dependent equation of state, one must be careful about the treatment of perturbations in dark energy when $w$ crosses -1 . We used the method provided in Refs. $[8,10]$ to treat the dark energy perturbations consistently in the whole parameter space in our numerical calculations. The sound speed of dark energy, $c_{s}^{2}$, is fixed in our calculation to be 1 ; note that the value of $c_{s}^{2}$ is actually insensitive to our final fit results. Our focus was put on the determinations of the spatial curvature of the observable universe, $\Omega_{k}$, and the total mass of neutrinos, $\sum m_{v}$, in our dynamical dark energy scenario, and the influence of these factors to the constraints on the dark energy parameters, $w_{0}$ and $w_{a}$.

The $95 \%$ CL limit on the spatial curvature is $-0.0153<$ $\Omega_{k}<0.0167$. Thus, we find that in our dynamical dark energy model the spatial curvature is well constrained by $\mathrm{WMAP}+\mathrm{LRG}+\mathrm{SN}+H_{0}$. In a curved universe, the constraint results of $w_{0}$ and $w_{a}$ are $w_{0}=-0.922 \pm 0.123$ and $w_{a}=$ $1.651 \pm 1.470(68 \% \mathrm{CL})$. Comparing to the case in a flat universe, $w_{0}=-0.921 \pm 0.098$ and $w_{a}=1.905 \pm 1.468(68 \%$ CL), we find that the error in $w_{0}$ is amplified by $25.51 \%$, and the error in $w_{a}$ is almost the same, only amplified by $0.14 \%$. Our global fit gives the constraint on the neutrino mass in our dynamical dark energy scenario, $\sum m_{v}<0.56 \mathrm{eV}(95 \%$ CL). So, we find that in our dynamical dark energy scenario the total mass of neutrinos can be well constrained by the data combination $\mathrm{WMAP}+\mathrm{LRG}+\mathrm{SN}+\mathrm{H}_{0}$. With the non-zero neutrino mass, the constraint results of $w_{0}$ and $w_{a}$ are $w_{0}=$ $-0.972 \pm 0.110$ and $w_{a}=2.038 \pm 1.492$ (68\% CL). Comparing to the case with a zero neutrino mass, $w_{0}=-0.921 \pm 0.098$ and $w_{a}=1.905 \pm 1.468(68 \% \mathrm{CL})$, we find that the error in $w_{0}$ is amplified by $12.24 \%$, and the error in $w_{a}$ is amplified by $1.63 \%$.

We believe that it is fairly important to use some divergence-free parametrization to probe the dynamical evolution of dark energy. We have shown that the form (1) is a good proposal, and it has been proven to be very successful in exploring the properties of dark energy. We suggest that this parametrization should be further investigated.

\section{Acknowledgments}

We acknowledge the use of the Legacy Archive for Microwave Background Data Analysis (LAMBDA). Support for LAMBDA is provided by the NASA Office of Space Science. The calculation is taken on Deepcomp7000 of Supercomputing Center, Computer Network Information Center of Chinese Academy of Sciences. This work was supported in part by the National Science Foundation of China under Grant Nos. 11175042, 10975032, 11033005 and 10705041, by the 973 program under Grant No. 2010CB83300, and by the National Ministry of Education of China under Grant Nos. NCET-090276 and N100505001. 
[1] M. Chevallier and D. Polarski, Int. J. Mod. Phys. D 10, 213 (2001) [arXiv:gr-qc/0009008]; E. V. Linder, Phys. Rev. Lett. 90, 091301 (2003) [arXiv:astro-ph/0208512].

[2] J. Z. Ma and X. Zhang, Phys. Lett. B 699, 233 (2011) [arXiv:1102.2671 [astro-ph.CO]].

[3] X. D. Li, S. Wang, Q. G. Huang, X. Zhang and M. Li, Sci. China-Phys. Mech. Astron. (2012), doi: 10.1007/s11433-0124748-z [arXiv:1202.4060 [astro-ph.CO]].

[4] H. Li and X. Zhang, Phys. Lett. B 703, 119 (2011) [arXiv:1106.5658 [astro-ph.CO]].

[5] Available at: http://cosmologist.info/cosmomc/; A. Lewis and S. Bridle, Phys. Rev. D 66, 103511 (2002).

[6] D. Spergel, et al., Astrophys. J. Suppl. 170 (2007) 377.

[7] J. Weller and A. Lewis, Mon. Not. R. Astron. Soc. 346 (2003) 987.

[8] J. Q. Xia, G. B. Zhao, B. Feng, H. Li, X. M. Zhang, Phys. Rev. D 73 (2006) 063521.

[9] B. Feng, M. Li, Y. S. Piao and X. M. Zhang, Phys. Lett. B 634, 101 (2006) [arXiv:astro-ph/0407432].

[10] G. B. Zhao, J. Q. Xia, M. Li, B. Feng and X. M. Zhang, Phys. Rev. D 72, 123515 (2005) [arXiv:astro-ph/0507482].

[11] J. Q. Xia, Y. F. Cai, T. T. Qiu, G. B. Zhao and X. Zhang, Int. J. Mod. Phys. D 17, 1229 (2008) [arXiv:astro-ph/0703202].

[12] H. Li and J. Q. Xia, JCAP 1004, 026 (2010) [arXiv:1004.2774 [astro-ph.CO]].

[13] E. Komatsu et al. [WMAP Collaboration], Astrophys. J. Suppl.
192, 18 (2011) [arXiv:1001.4538 [astro-ph.CO]].

[14] Available at: http://lambda.gsfc.nasa.gov/.

[15] K. N. Abazajian et al. [SDSS Collaboration], Astrophys. J. Suppl. 182, 543 (2009) [arXiv:0812.0649 [astro-ph]].

[16] A. G. Riess et al., Astrophys. J. 730, 119 (2011) [Erratum-ibid. 732, 129 (2011)] [arXiv:1103.2976 [astro-ph.CO]].

[17] E. Di Pietro and J. F. Claeskens, Mon. Not. Roy. Astron. Soc. 341, 1299 (2003).

[18] T. M. Davis et al., Astrophys. J. 666, 716 (2007) [astro$\mathrm{ph} / 0701510]$.

[19] M. Li, X. D. Li and X. Zhang, Sci. China Phys. Mech. Astron. 53, 1631 (2010) [arXiv:0912.3988 [astro-ph.CO]].

[20] K. S. Hirata et al., Phys. Lett. B 280, 146 (1992); Y. Fukuda et al., Phys. Lett. B 335, 237 (1994); Y. Fukuda et al., Phys. Rev. Lett. 81, 1562 (1998); W. W. M. Allison et al., Phys. Lett. B 449, 137 (1999); M. Ambrosio et al., Phys. Lett. B 517, 59 (2001).

[21] B. T. Cleveland et al., Astrophys. J. 496, 505 (1998); W. Hampel et al., Phys. Lett. B 447, 127 (1999); J. N. Abdurashitov et al., Phys. Rev. C 60, 055801 (1999); S. Fukuda et al., Phys. Rev. Lett. 86, 5651 (2001); S. Fukuda et al., Phys. Rev. Lett. 86, 5656 (2001); Q. R. Ahmad et al., Phys. Rev. Lett. 89, 011301 (2002); S. N. Ahmed et al., Phys. Rev. Lett. 92, 181301 (2004).

[22] A. D. Dolgov, Phys. Rept. 370, 333 (2002); J. Lesgourgues and S. Pastor, Phys. Rept. 429, 307 (2006). 